\title{
GRO J1744-28 AND THE RAPID BURSTER; BIZARRE OBJECTS!
}

\author{
WALTER H.G. LEWIN \\ MIT \\ Cambridge, $M A$
}

The bursts from GRO J1744-28 are due to accretion instabilities as is the case for type II bursts in the Rapid Burster. Both sources are transient Low-Mass X-ray Binaries, and they both exhibit unusual quasi-periodicoscillations in their persistent X-ray flux following several (not all) of the type II bursts. There are important differences too. GRO J1744-28 is an Xray pulsar; the Rapid Burster is not. In addition, the pattern of bursts and the burst peak luminosities are very different for the two sources. Time intervals between the rapidly repetitive bursts in the Rapid Burster can be as short as $10 \mathrm{sec}$, in $1744-28$ they are as short as $200 \mathrm{sec}$. The peak luminosities of the bursts from GRO J1744-28 can exceed the Eddington luminosity (for assumed isotropic emission) by one to two orders of magnitude. The QPO centroid frequencies (see above) differ by an order of magnitude ( $\sim 04 \mathrm{~Hz}$ for the Rapid Burster, and $0.3 \mathrm{~Hz}$ for GRO J1744-28). The difference in behavior $\mathrm{p}$ obably lies in the difference in the magnetic dipole field strength of the accreting neutron stars (for GRO J1744-28 it is almost certainly much higher than for the Rapid Burster). It remains puzzling, why GRO J1744-28 and the Rapid Burster are the only known sources which exhibit rapidly repetitive type II bursts.

Unlike GRO J1744-28, the Rapid Burster also emits type I bursts. No $\mathrm{kHz}$ near-coherent oscillations have been found in a handful of type I bursts from the Rapid Burster, observed with RXTE. However, $\mathrm{kHz}$ oscillations have been observed in several type II bursts from the Rapid Burster. For more details see Guerriero, Lewin, and Kommers, IAUC 6689, (1997); also Guerriero et al., manuscript in preparation.

The following papers compare in some detail GRO J1744-28 with the Rapid Burster: Lewin et al., ApJ 462, L39 (1996) and Kommers et al., ApJ 482, L53 (1997). 